\title{
Cerium oxide nanoparticle administration to skeletal muscle cells under different gravity and radiation conditions
}

Giada Graziana Genchi ${ }^{a,{ }^{*}, *}$, Andrea Degl'Innocenti ${ }^{a,{ }^{*}, *}$, Chiara Martinelli ${ }^{a,{ }^{*}, *}$, Matteo Battaglini $^{a}$, Daniele De Pasquale ${ }^{a, b}$, Mirko Prato $^{c}$, Sergio Marras ${ }^{c}$, Giammarino Pugliese $^{d}$, Filippo Drago ${ }^{d}$, Alessandro Mariani ${ }^{e}$, Michele Balsamo ${ }^{e}$, Valfredo Zolesi $^{e}$, Gianni Ciofani ${ }^{a}{ }^{*}$

${ }^{a}$ Istituto Italiano di Tecnologia, Smart Bio-Interfaces, Viale Rinaldo Piaggio 34, 56025 Pontedera (Pisa), Italy

bScuola Superiore Sant'Anna, The BioRobotics Institute, Viale Rinaldo Piaggio 34, 56025 Pontedera (Pisa), Italy

${ }^{c}$ Istituto Italiano di Tecnologia, Materials Characterization, Via Morego 20, 16163 Genova, Italy ${ }^{\mathrm{d}}$ Istituto Italiano di Tecnologia, Nanochemistry, Via Morego 20, 16163 Genova, Italy ${ }^{\mathrm{e} K a y s e r ~ I t a l i a ~ S . r .1 ., ~ V i a ~ d i ~ P o p o g n a ~ 501, ~} 57128$ Livorno, Italy 
(A)

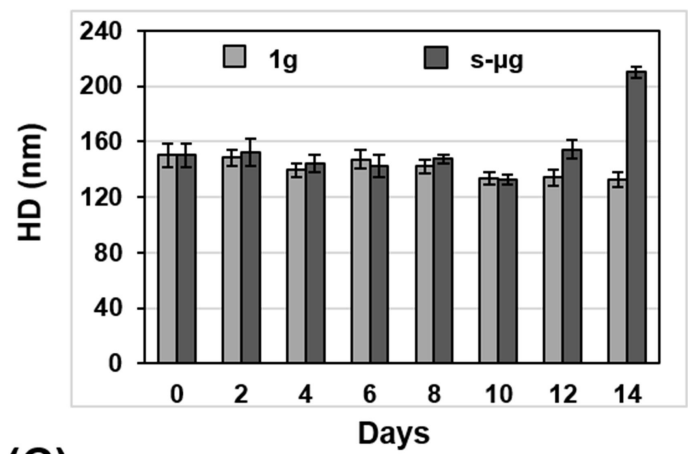

(C)

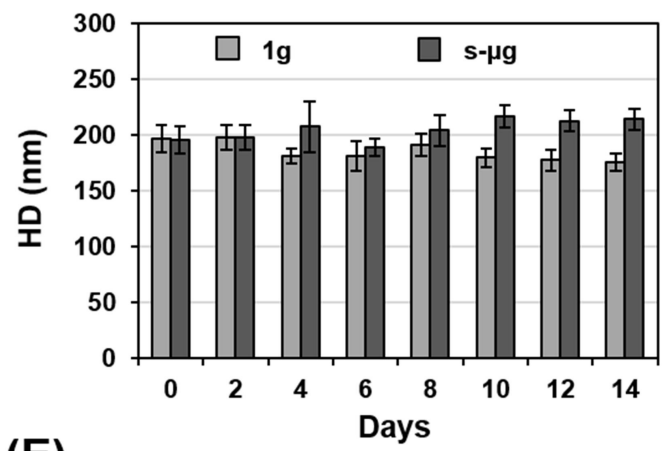

(E)

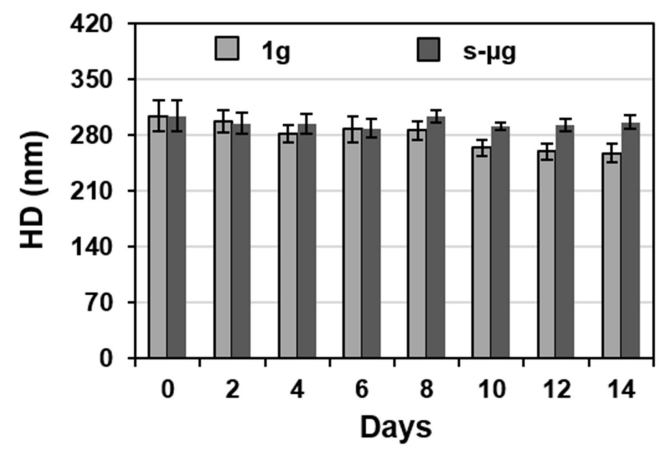

(B)

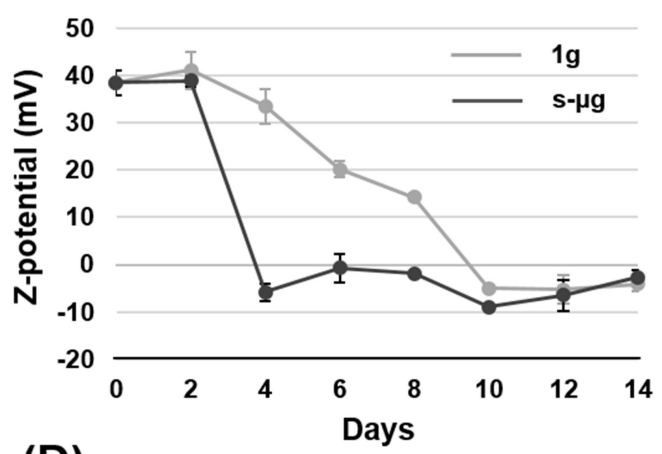

(D)

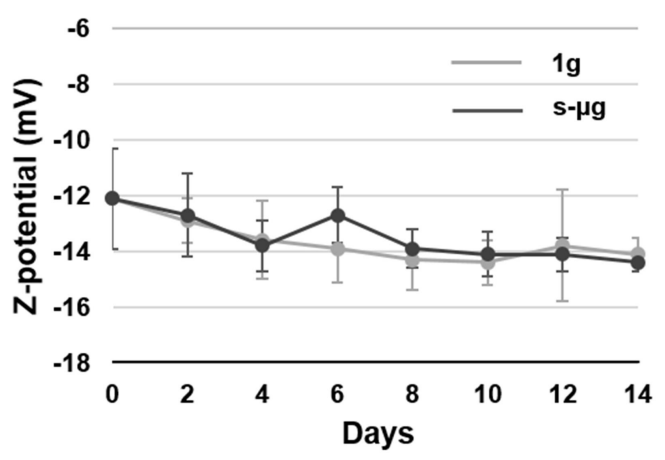

Figure S1. Characterization of cerium oxide nanoparticle (NC) dispersions. (A) Dynamic light scattering (DLS) analysis of $\mathrm{NC}$ dispersions in ultrapure water after exposure to normal gravity and to simulated microgravity. (B) Zpotential measurements of $\mathrm{NC}$ dispersions in ultrapure water after exposure to normal gravity and to simulated microgravity. (C) DLS analysis of FBS-coated NC dispersions in ultrapure water after exposure to normal gravity and to simulated microgravity. (D) Z-potential measurements of fetal bovine serum (FBS)-coated NC dispersions in ultrapure water after exposure to normal gravity and to simulated microgravity. (E) DLS analysis of FBS-coated NC dispersions in $10 \%$ FBS solution in ultrapure water after exposure to normal gravity and to simulated microgravity. "HD" stands for hydrodynamic diameter, and "s- $\mu \mathrm{g}$ " stands for simulated microgravity. 


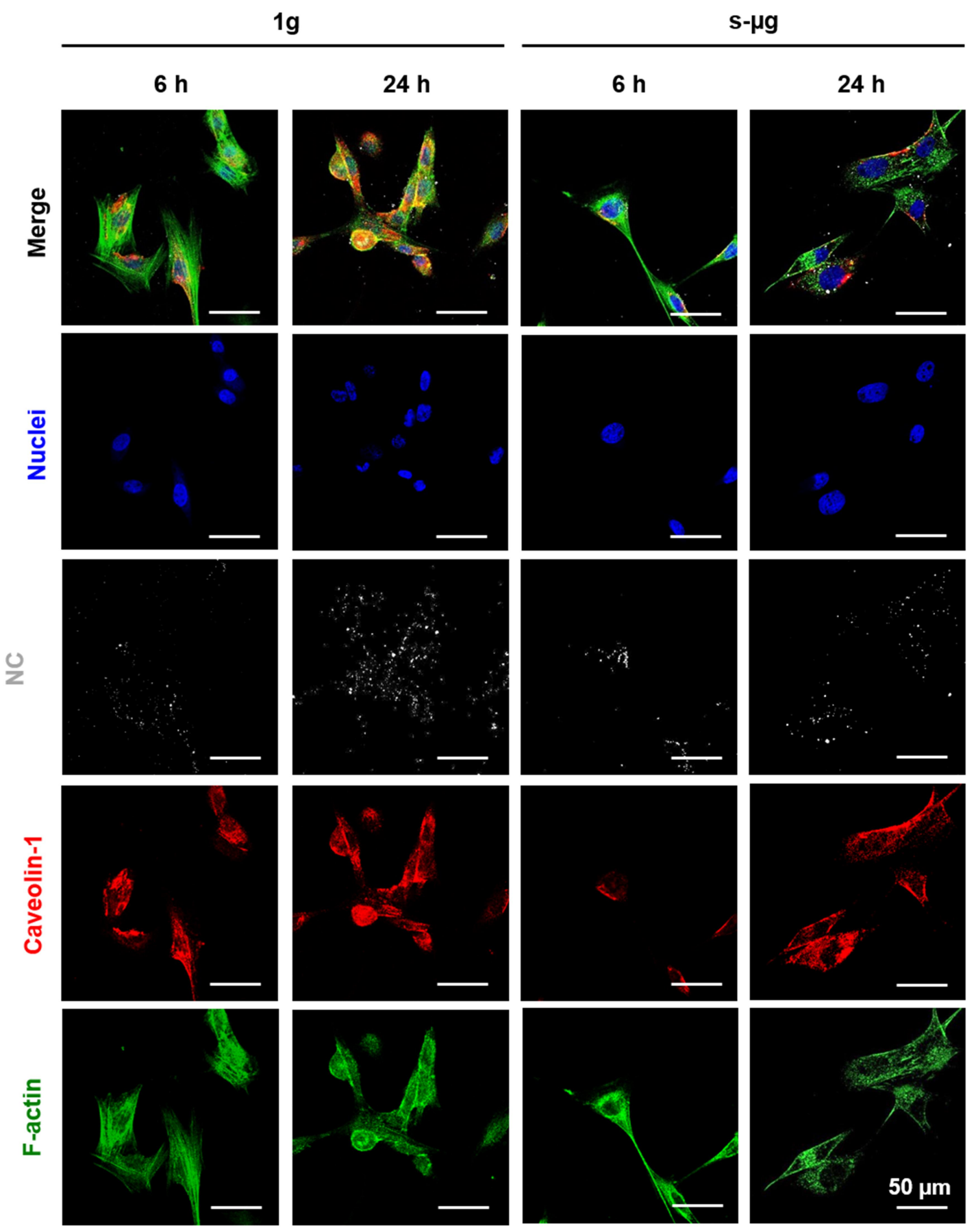

Figure S2. Internalization study through immunofluorescence staining of caveolin-1 and confocal microscopy imaging. Nanoparticle signal originates from light scattering. "NC" stands for nanoceria; "s- $\mu \mathrm{g}$ " stands for simulated microgravity. 
19
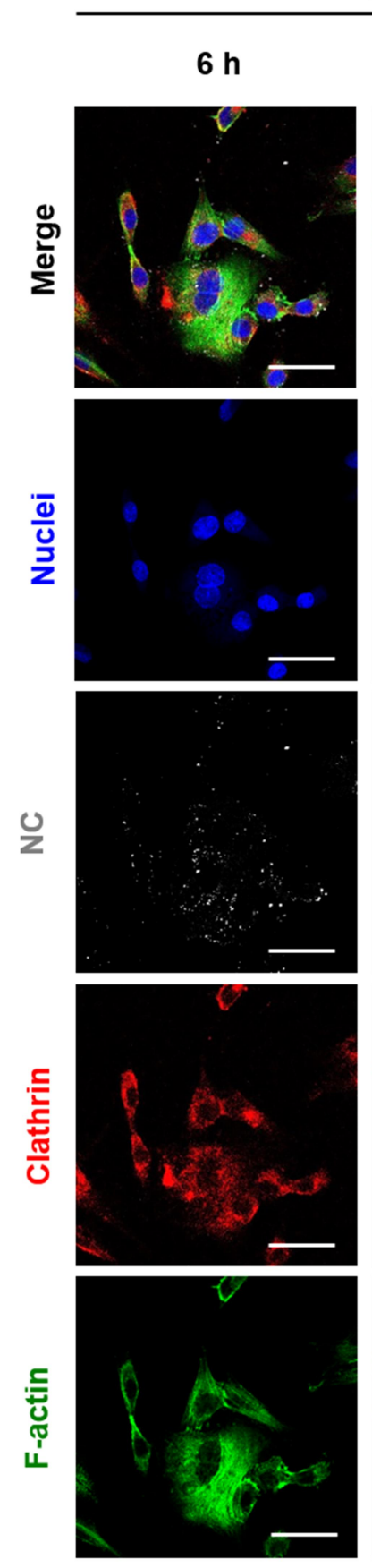

s- $\mu \mathrm{g}$
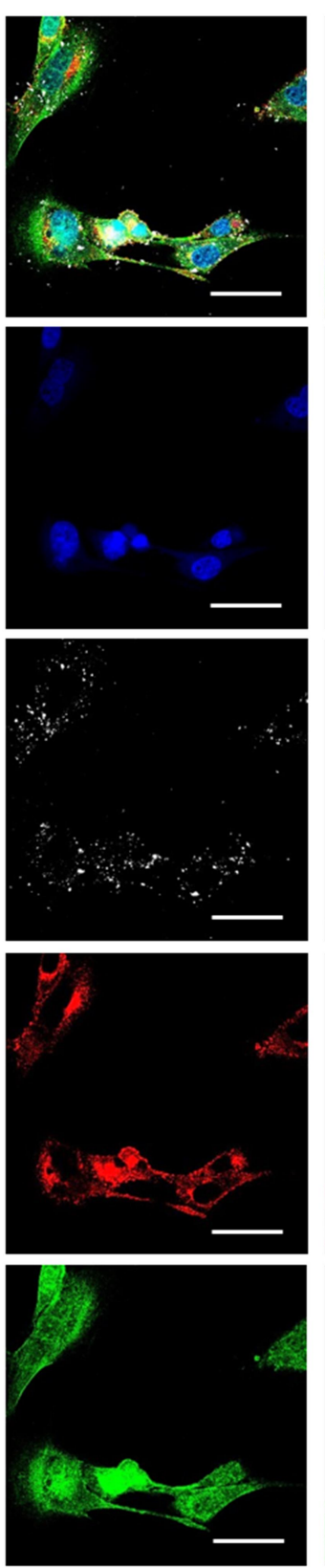

$6 \mathrm{~h}$
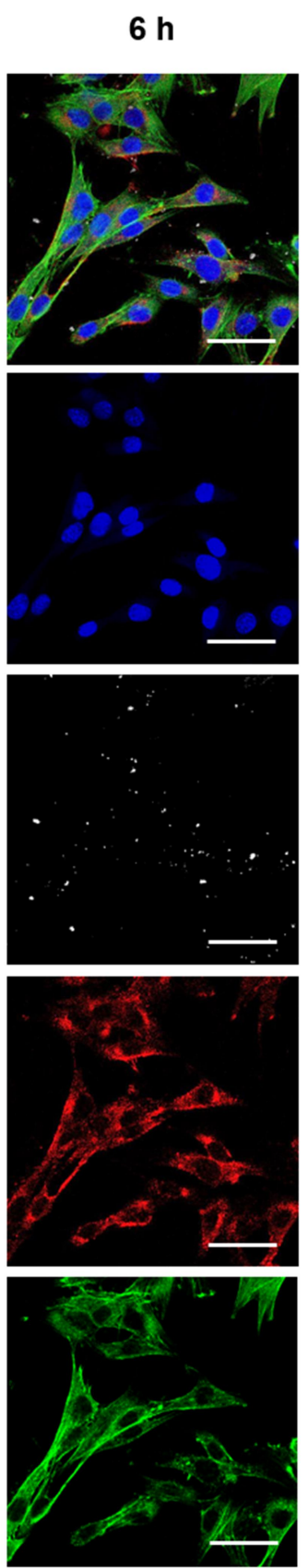

$24 \mathrm{~h}$
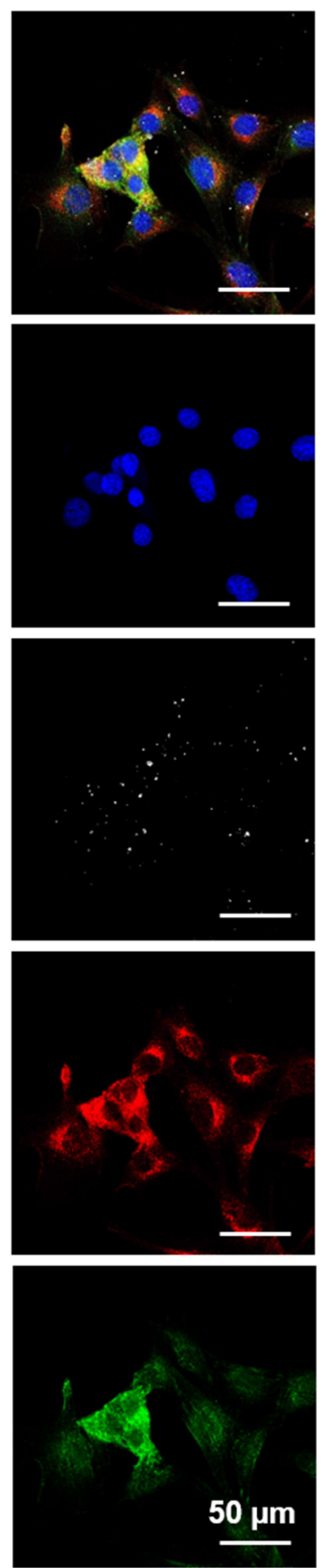

Figure S3. Internalization study through immunofluorescence staining of clathrin and confocal microscopy imaging. Nanoparticle signal originates from light scattering. "NC" stands for nanoceria; "s- $\mu \mathrm{g}$ " stands for simulated microgravity. 

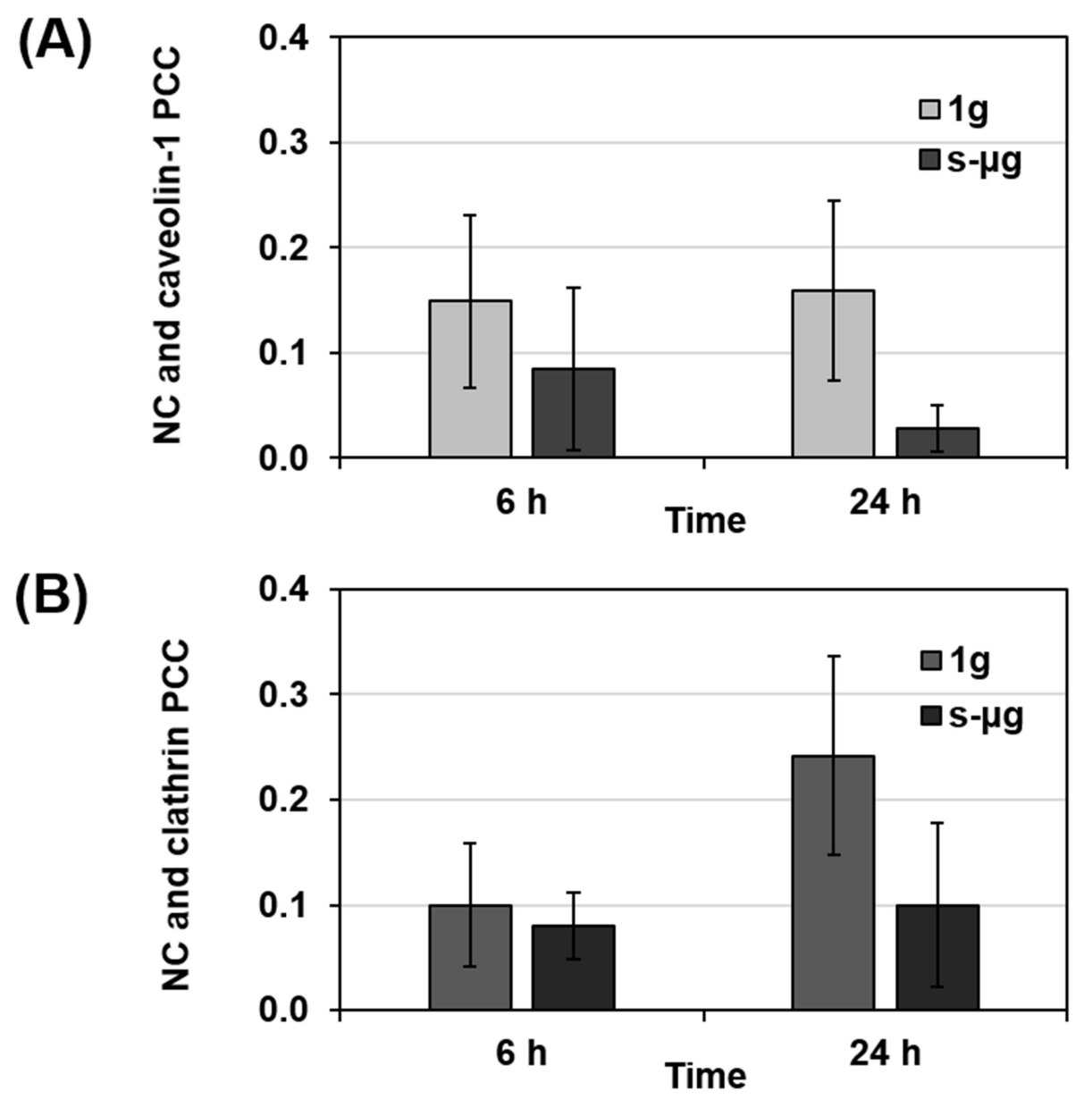

Figure S4: Internalization study. (A) Histograms depicting Pearson's correlation coefficient (PCC) measuring co-localization of signal from nanoceria (NC) and caveolin-1 in cell cultures, exposed to either normal gravity or simulated microgravity for $6 \mathrm{~h}$ and $24 \mathrm{~h}$. (B) Histograms depicting PCC measuring co-localization of signal from $\mathrm{NC}$ and clathrin in cell cultures exposed either to normal gravity and to simulated microgravity for $6 \mathrm{~h}$ and $24 \mathrm{~h}$. "s$\mu \mathrm{g}$ ” stands for simulated microgravity; “+NC” stands for nanoceria-treated; "-NC” stands for non-nanoceria-treated. Data are represented as average \pm standard deviation $(n=3)$. 


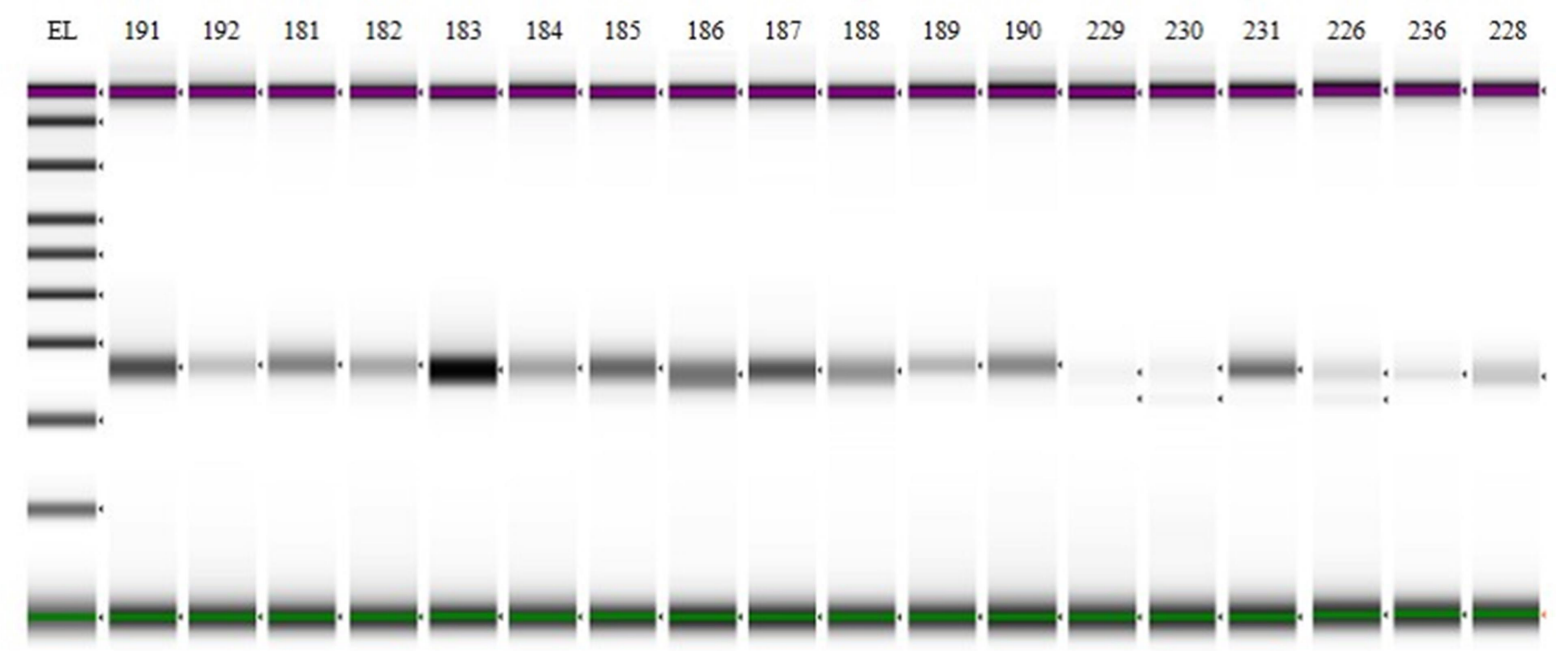

Figure S5. RNA quality assessment obtained by Bioanalyzer (Agilent). "EL" stands for electronic ladder. Numbers indicate each experiment unit. 
Table S1. Photometric analysis of total RNA after purification.

\begin{tabular}{|c|c|c|c|c|c|c|c|c|}
\hline Place & $\mathbf{E U}$ & Treatment & $\begin{array}{l}\text { Gravity } \\
\text { regimen }\end{array}$ & $\begin{array}{l}\text { RNA } \\
(\mathrm{ng} / \mu \mathrm{l})\end{array}$ & $\mathbf{A 2 6 0}$ & A280 & $\begin{array}{l}\text { A260/ } \\
\text { A280 }\end{array}$ & $\begin{array}{l}\text { A260/ } \\
\text { A230 }\end{array}$ \\
\hline ISS & 191 & $-\mathrm{NC} 1$ & $\mu \mathrm{g}$ & 23.6 & 0.591 & 0.299 & 1.98 & 0.35 \\
\hline ISS & 192 & $-\mathrm{NC} 2$ & $\mu g$ & 12.2 & 0.304 & 0.152 & 2.00 & 0.15 \\
\hline ISS & 181 & $-\mathrm{NC} 3$ & $\mu \mathrm{g}$ & 9.6 & 0.240 & 0.125 & 1.92 & 0.11 \\
\hline ISS & 182 & $+\mathrm{NC} 1$ & $\mu \mathrm{g}$ & 13.6 & 0.341 & 0.187 & 1.82 & 0.73 \\
\hline ISS & 183 & $+\mathrm{NC} 2$ & $\mu \mathrm{g}$ & 9.3 & 0.231 & 0.123 & 1.88 & 0.41 \\
\hline ISS & 184 & $+\mathrm{NC} 3$ & $\mu \mathrm{g}$ & 5.9 & 0.148 & 0.085 & 1.74 & 0.12 \\
\hline ISS & 185 & $-\mathrm{NC} 1$ & $1 \mathrm{~g}$ & 21.8 & 0.544 & 0.283 & 1.93 & 0.48 \\
\hline ISS & 186 & $-\mathrm{NC} 2$ & $1 \mathrm{~g}$ & 20.5 & 0.513 & 0.248 & 2.07 & 0.18 \\
\hline ISS & 187 & $-\mathrm{NC} 3$ & $1 g$ & 9.9 & 0.247 & 0.131 & 1.89 & 0.27 \\
\hline ISS & 188 & $+\mathrm{NC} 1$ & $1 \mathrm{~g}$ & 10.4 & 0.260 & 0.134 & 1.95 & 0.20 \\
\hline ISS & 189 & $+\mathrm{NC} 2$ & $1 \mathrm{~g}$ & 14.1 & 0.353 & 0.154 & 2.29 & 0.17 \\
\hline ISS & 190 & $+\mathrm{NC} 3$ & $1 \mathrm{~g}$ & 17.4 & 0.435 & 0.202 & 2.15 & 0.17 \\
\hline IIT & 229 & $-\mathrm{NC} 1$ & $1 \mathrm{~g}$ & 7.6 & 0.190 & 0.089 & 2.14 & 0.09 \\
\hline IIT & 230 & $-\mathrm{NC} 2$ & $1 \mathrm{~g}$ & 5.4 & 0.135 & 0.077 & 1.76 & 0.19 \\
\hline IIT & 231 & -NC3 & $1 g$ & 9.0 & 0.225 & 0.144 & 1.56 & 0.35 \\
\hline IIT & 226 & $+\mathrm{NC} 1$ & $1 \mathrm{~g}$ & 4.8 & 0.119 & 0.083 & 1.44 & 0.25 \\
\hline IIT & 236 & $+\mathrm{NC} 2$ & $1 \mathrm{~g}$ & 5.2 & 0.130 & 0.084 & 1.55 & 0.05 \\
\hline IIT & 228 & $+\mathrm{NC} 3$ & $1 \mathrm{~g}$ & 5.1 & 0.127 & 0.083 & 1.53 & 0.37 \\
\hline
\end{tabular}

"A" stands for absorbance at either 260 (A260) or 280 (A280) nm; "EU" stands for experiment unit. "IIT" stands for Istituto Italiano di Tecnologia; "ISS" stands for International Space Station. The string defining sample treatment starts with a notation indicating whether a sample has been treated with nanoceria (+NC) or not (-NC), followed by the replicate number. For instance, "+NC1” means nanoceria-treated replicate 1. 